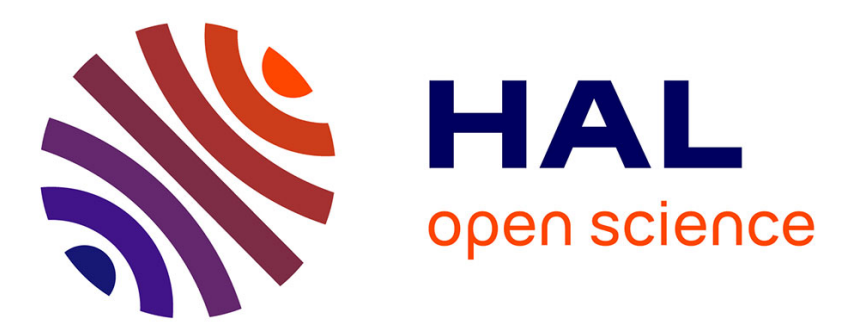

\title{
Unifying Impacts in Granular Matter from Quicksand to Cornstarch
}

\author{
J. John Soundar Jerome, Nicolas Vandenberghe, Yoël Forterre
}

\section{To cite this version:}

J. John Soundar Jerome, Nicolas Vandenberghe, Yoël Forterre. Unifying Impacts in Granular Matter from Quicksand to Cornstarch. Physical Review Letters, 2016, 117 (9), 10.1103/PhysRevLett.117.098003 . hal-01431977

\section{HAL Id: hal-01431977 https://hal.science/hal-01431977}

Submitted on 11 Jan 2017

HAL is a multi-disciplinary open access archive for the deposit and dissemination of scientific research documents, whether they are published or not. The documents may come from teaching and research institutions in France or abroad, or from public or private research centers.
L'archive ouverte pluridisciplinaire HAL, est destinée au dépôt et à la diffusion de documents scientifiques de niveau recherche, publiés ou non, émanant des établissements d'enseignement et de recherche français ou étrangers, des laboratoires publics ou privés. 


\title{
Unifying Impacts in Granular Matter from Quicksand to Cornstarch
}

\author{
J. John Soundar Jerome, ${ }^{1,2, \dagger}$ Nicolas Vandenberghe, ${ }^{2}$ and Yoël Forterre ${ }^{1, *}$ \\ ${ }^{1}$ Aix Marseille Univ, CNRS, IUSTI, 13013 Marseille, France \\ ${ }^{2}$ Aix Marseille Univ, CNRS, Centrale Marseille, IRPHE, 13013 Marseille, France
}

(Received 15 March 2016; published 23 August 2016)

\begin{abstract}
A sharp transition between liquefaction and transient solidification is observed during impact on a granular suspension depending on the initial packing fraction. We demonstrate, via high-speed pressure measurements and a two-phase modeling, that this transition is controlled by a coupling between the granular pile dilatancy and the interstitial fluid pressure generated by the impact. Our results provide a generic mechanism for explaining the wide variety of impact responses in particulate media, from dry quicksand in powders to impact hardening in shear-thickening suspensions like cornstarch.
\end{abstract}

DOI: 10.1103/PhysRevLett.117.098003

Impacts on particulate media like granular materials and suspensions present an astonishingly rich phenomenology [1,2]. Along with its astrophysical [3] and ballistics applications [4], impact dynamics is an object of active research to understand the high-speed response of granular matter [5]. In dry granular media, impact by a solid object results in the formation of a corona of granular ejecta and a solidlike plastic deformation leading to a permanent crater [6-11]. For fine powders in air, granular jets and cavity collapse occur during impact $[12,13]$. Subsequent studies showed that the ambient pressure of the interstitial fluid (air) is an important element for the observed fluidlike behavior [14-16], while for denser packing the impact penetration is much reduced [17]. However, the question of the physical mechanisms and control parameters that give rise to such a wide variety of phenomena is still largely open. Recently, studies on shear-thickening suspensions (cornstarch) showed another kind of behavior. Above a critical velocity, an impacting object immediately stops [18], or in some cases generates cracks [19], as if hitting a solid. This phenomenon has been related to the propagation of dynamic jamming fronts in the bulk [18]. However, the arrest mechanism remains unclear and explanations so far overlook the role of the coupling between the interstitial fluid and the grains, which can strongly affect the transient behavior of saturated granular materials [20-22]. Understanding the origin of impact-activated solidification in these systems should help to clarify the physics of shearthickening fluids-a still debated topic [23-27].

The objective of this Letter is to address these issues and elucidate the role of the interstitial fluid and the initial volume fraction on the impact dynamics in dense suspensions. To avoid difficulties associated with colloidal interactions between particles (like in shear-thickening suspensions) or fluid compressibility (like in powders in air), we study here the impact of a freely falling rigid sphere on a model granular suspension [28] made up of macroscopic, heavy particles (glass beads in the range $0.1-1 \mathrm{~mm}$ ) immersed in an incompressible fluid (water, viscous oil). The initial packing fraction of the suspension $\phi_{0}$ (the ratio of the volume of the glass beads to the total volume) is controlled by first fluidizing the mixture and then compacting the sediment, before removing the excess liquid [see Fig. 1(a) and the Supplemental Material [29]]. For a granular pile of frictional spherical particles, $\phi_{0}$ typically takes values between 0.55 and 0.62 [22]. The suspension is kept fully saturated so that surface tension effects can be neglected.

Remarkably, two very distinct impact regimes are observed depending on the initial packing fraction [Figs. 1(b), $1(\mathrm{c})]$. For initially loose packing $\left(\phi_{0}=0.560\right)$, the ball readily sinks in the suspension, giving rise to a collapsing cavity and a central jet (see movie 1 in the Supplemental Material [29]). Such fluidlike behavior is strikingly similar to that observed when a rigid sphere hits a dry loose powder under atmospheric pressure [12,13]. By contrast, for dense packing $\left(\phi_{0}=0.604\right)$, the ball stops abruptly within a few milliseconds as it hits the surface (see movie 2 in the Supplemental Material [29]). This solidlike behavior is reminiscent of the impact-activated solidification observed in a shear-thickening suspension like cornstarch [18]. It strongly depends on the grain size and viscosity of the interstitial fluid between the particles. Using coarser particles in the same fluid tends to suppress the extreme deceleration, which can be restored by increasing the fluid viscosity (see movie 3 in the Supplemental Material [29]).

To explain such a drastic change between the impact behavior of the loose and dense suspension, we rely on a pore-pressure feedback mechanism: a coupling between the deformation of the granular medium and the pressure of the interstitial fluid between the grains [20,21]. As first described by Reynolds [30], when a dense granular packing starts to flow, it must dilate. Since the medium is saturated with an incompressible fluid, the fluid is sucked in, as evidenced by the bright zone developing beneath the impacting sphere in the dense case [Fig. 1(b)]. Therefore, when the ball hits the pile, the interstitial fluid pressure (pore-pressure) drops 
(a)
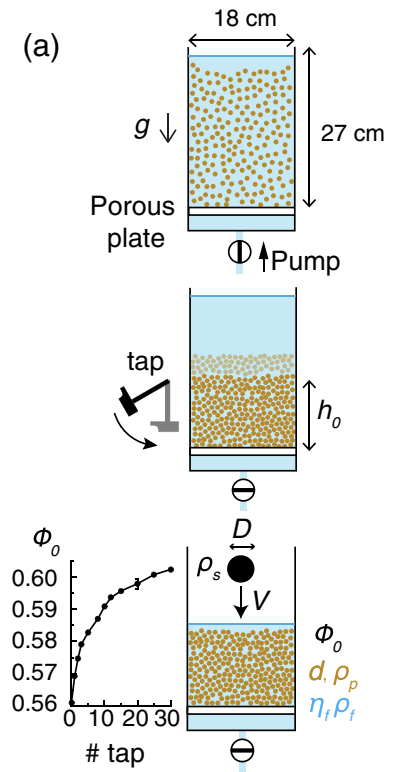
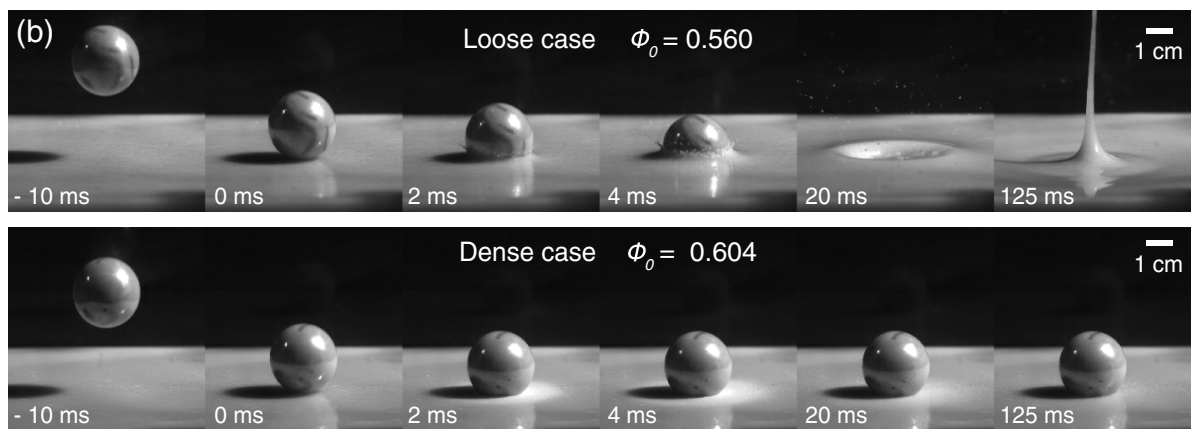

(c)

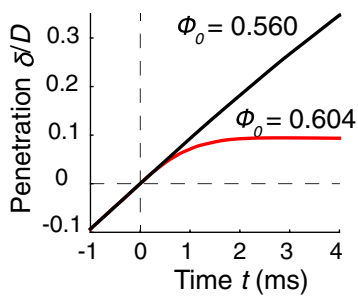

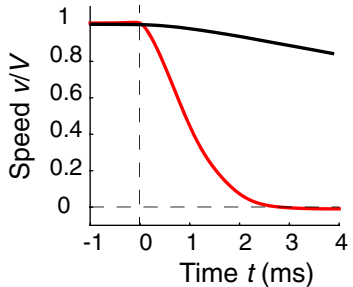

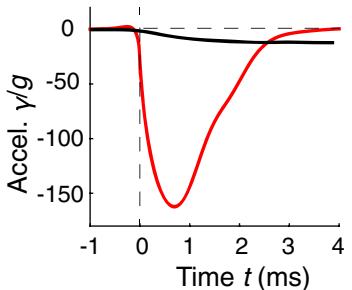

FIG. 1. Liquid-solid transition after impact on a suspension of heavy particles (glass beads of density $\rho_{p}=2500 \mathrm{~kg} / \mathrm{m}^{3}$ and diameter $d=170 \mu \mathrm{m}$ in water). (a) Protocol used to prepare the nonbuoyant suspension at a given initial packing fraction $\phi_{0}\left(h_{0}=9-10 \mathrm{~cm}\right.$ depending on the number of taps). (b) Image sequence of a solid sphere (glass marble of diameter $D=25.2 \mathrm{~mm}$, density $\rho_{s}=2500 \mathrm{~kg} / \mathrm{m}^{3}$, speed $V=2.35 \mathrm{~m} / \mathrm{s}$ ) impacting the suspension in the loose (top) or dense (bottom) case. (c) Corresponding penetration, speed, and acceleration of the impacting sphere as a function of time. Time $t=0$ gives the instant when the sphere hits the granular bed surface.

instantaneously, which in turn presses the grains against each other, thereby enhancing the friction. Thus, the medium is transiently solidified. A loosely packed granular bed, on the other hand, tends to compact when it deforms. Therefore, a rise in pore pressure is produced during impact that can balance the weight of the grains. This suppresses the contact network, resulting in local or global fluidization.

We proceed a direct verification of this mechanism by developing a high-frequency measurement of the interstitial fluid pressure inside the suspension, just under the impact [Fig. 2(a)] (see Fig. S1 and the Supplemental Material [29]). In the loose case [Fig. 2(b), upper panel], the pore pressure shows a sudden positive peak after impact. The measured peak pressure $(\sim 1 \mathrm{kPa})$ is of the order of the effective weight of the suspension above the sensor [31], indicating a fluidization of the medium. At longer times sedimentation proceeds and the pressure slowly relaxes to zero. By contrast, in the dense case [Fig. 2(b) lower panel], the peak pressure is negative and its magnitude $(10-100 \mathrm{kPa})$ is much higher than the confining pressure due to gravity, indicating that particles are strongly pressed against each other, effectively leading to a solidification of the medium. Interestingly, the perturbation triggered by the impact remains localized near the impact point [inset of lower panel of Fig. 2(b)]. The transition between a positive and negative peak pressure occurs for a critical packing fraction $\phi_{c}=0.585 \pm 0.0053$, which is independent of the projectile diameter $D$ and impact speed $V$ [Fig. 2(c)]. This value is consistent with previous rheological measurements (a)

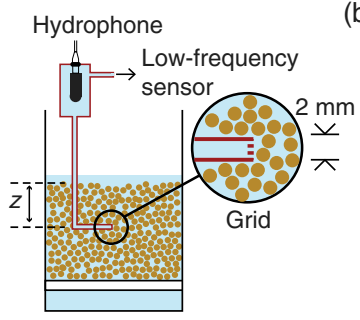

(c)

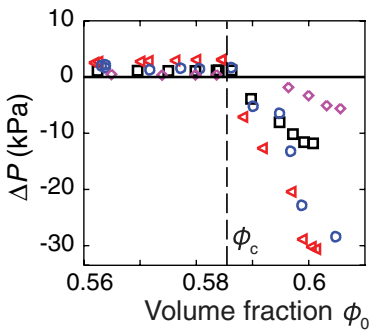

(b)

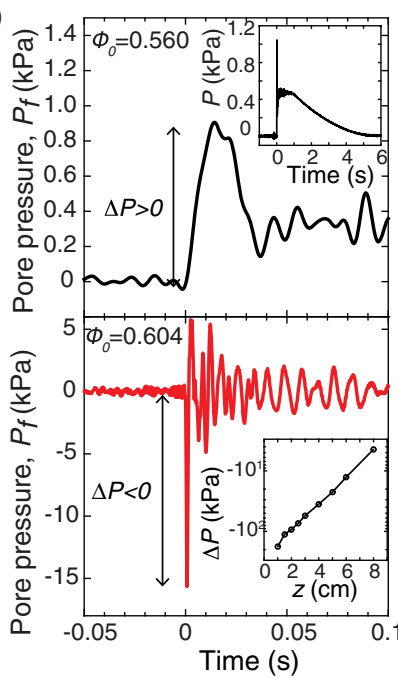

FIG. 2. (a) Experimental setup to measure pore pressure at different heights $z$ in the suspension. (b) Pore-pressure signals in the loose case (upper panel) and dense case (lower panel) (same experimental conditions as in Fig. $1, z=6 \mathrm{~cm}$ ). In the dense case, the negative peak pressure intensity decreases with the distance from the impact (inset). (c) Peak pore pressure as function of the initial packing fraction $\phi_{0}$ for various impact speeds $V$, sphere diameters $D$, and sensor positions $z$ (diamonds: $V=1.0 \mathrm{~m} / \mathrm{s}, D=16.5 \mathrm{~mm}, z=2 \mathrm{~cm}$; circles: $V=3.4 \mathrm{~m} / \mathrm{s}$, $D=16.5 \mathrm{~mm}, z=2 \mathrm{~cm}$; square: $V=2.4 \mathrm{~m} / \mathrm{s}, D=25.2 \mathrm{~mm}$, $z=5 \mathrm{~cm}$; triangles: $V=4.8 \mathrm{~m} / \mathrm{s}, D=25.2 \mathrm{~mm}, z=5 \mathrm{~cm}$ ). 
of the jamming packing fraction using frictional spherical particles [28].

To model the impact dynamics, the coupling between the grain matrix deformation and the interstitial fluid should necessarily be taken into account. The Darcy-Reynolds equation

$$
\nabla^{2} P_{f}=\frac{\eta_{f}}{\kappa} \alpha \dot{\gamma}\left(\phi-\phi_{c}\right),
$$

is obtained by coupling Darcy law $[32,33]$ for the fluid flow in a medium of permeability $\kappa \propto d^{2}$ and fluid viscosity $\eta_{f}$ and an empirical equation for Reynolds dilatancy that accounts for the change of volume fraction with the shear at rate $\dot{\gamma}[22,34,35]$, where $\alpha$ is a constant of order unity (see Supplemental Material [29] for the derivation of the equation). This is a Poisson-type equation for the pore pressure $P_{f}$, wherein the sign of the source term is imposed only by $\phi-\phi_{c}$. Equation (1) implies that the pore-pressure generated by the impact scales as

$$
P_{f} \sim-\frac{\eta_{f}}{\kappa} \alpha \Delta \phi V_{p} L,
$$

where $\Delta \phi=\phi_{0}-\phi_{c}, V_{p}$ is the velocity scale for the particles velocity field, $L$ the typical extent up to which deformation is experienced by the granular bed, and $\alpha$ a constant of order unity. This relation predicts that the pore pressure is positive (fluidlike response) or negative (solidlike response) depending only on the sign of $\Delta \phi$, while its magnitude is controlled by both the grain diameter and fluid viscosity, in agreement with our observations. We note that the Reynolds dilatancy equation [and thus Eq. (2)] is valid as long as the contact pressure between grains remains positive (since the grains are noncohesive). When fluidization occurs in the loose state, the contact network vanishes and the pore pressure saturates to the effective weight of the suspension, which explains the strong dissymmetry in Fig. 2(c).

The Darcy-Reynolds model (2) can be used to quantitatively infer the penetration dynamics in the dense case $(\Delta \phi>0)$, where the Reynolds equation is always valid. Assuming a frictional rheology for the granular suspension [22] and neglecting the confining pressure due to gravity as compared to the pore pressure, the contact stress on the impactor is $-A P_{f}$, where $A$ is an effective friction coefficient $[36,37]$ and $P_{f}$ is the pore pressure (2), in which $V_{p}=\dot{\delta}$, where $\delta$ is the penetration depth, and $L=a$ the typical radius of the contact area of the projectile [consistent with our measurements of the pressure profile; see inset of lower panel of Fig. 2(b)]. Using Newton's second law for the impactor $\rho_{s}\left(\pi D^{3} / 6\right) \ddot{\delta}=\pi a^{2} A P_{f}$, where $\rho_{s}$ is the density of the projectile, and assuming small penetration $\left(a^{2} \approx D \delta\right)$, the penetration $\delta$ then evolves according to the nondimensional equation [after integration with initial conditions $\delta(0)=0$ and $\dot{\delta}(0)=V]: d \tilde{\delta} / \tilde{d} \tilde{t}=-(2 / 5) \tilde{\delta}^{5 / 2}+1$, where $\tilde{\delta}=\delta /\left(V t_{m}\right)$ is the dimensionless penetration and $\tilde{t}=t / t_{m}$ a dimensionless time given by

$$
t_{m}=\frac{D}{V}(\lambda \Delta \phi)^{-2 / 5}, \quad \text { where } \lambda=6 A \alpha \frac{\eta_{f} D}{\rho_{s} \kappa V} .
$$

This dynamics is compared with experiments at various initial packing fraction $\phi_{0}>\phi_{c}$ for a given projectile and impact velocity in Fig. S2 (see Supplemental Material [29]). As $\phi_{0}$ approaches $\phi_{c}$, the indentation depth increases and the maximal deceleration decreases, while the stopping time increases. A collapse of all trajectories is obtained when the variables are rescaled according to the model prediction (see Fig. S2). Quantitative agreement is achieved by taking $A \alpha \simeq 30$. Such a value is consistent with experimental measurements of $\alpha$ and $A$ for glass beads [21,37], for which $\alpha \approx 2-4$ and $A \approx 5-15$. Furthermore, the Darcy-Reynolds model captures the impact dynamics for a wide range of physical parameters: using different suspension mixtures and different impact parameters, the data for the maximal indentation depth $\delta_{\max }$, stopping time $t_{\text {end }}$ and maximal deceleration $\gamma_{\max }$ collapse on the model when plotted as function of $\lambda \Delta \phi$ [Figs. 3(a), 3(b)]. The slope of the scaling law (thick dashed line) is nevertheless weaker than in experiments. Accounting for the exact geometrical relationship between the indentation depth and the contact area, and the dependence of $A$ with the initial volume fraction, give a better trend (thin dashed lines, see Supplemental Material [29]). Finally, we systematically measure the pore-pressure profile below the impact point for different impactor parameters and suspensions [Fig. 3(c)]. A universal exponential decay is obtained when the pore pressure is scaled by the maximal pore pressure given by the Darcy-Reynolds scaling (2) and the depth $z$ is scaled by $L=a \sim \sqrt{D V t_{m}}$, thereby conclusively supporting the model.

These results can be extended to suspensions for which the interstitial fluid is not a liquid but a gas, like dry powders at different operating air pressure. As long as the diffusion time scale for gas expulsion $\tau_{d} \sim \eta \chi L^{2} / \kappa$, where $\chi$ is the gas compressibility, is small compared to the impact time scale $\tau_{i} \sim D / V$, the fluid can be assumed incompressible and the Darcy-Reynolds scaling (2) of the pore pressure still holds (see Supplemental Material [29]). Otherwise, the fluid has no time to escape from the pores during impact. The pore pressure is then given by a gas state equation $P_{f} \sim-(1 / \chi) \Delta \phi /\left[\phi_{0}\left(1-\phi_{0}\right)\right]$, where $\chi \propto 1 / P_{0}$ and $P_{0}$ is the gas pressure. In Fig. 4, we compare the predicted pore pressure $P_{f}$ with the typical confining pressure due to gravity $P_{\text {grav }} \sim \phi_{0} \Delta \rho g D$ for our study and previous impact studies covering a wide range of particulate media and impact conditions, and for both incompressible and compressible interstitial fluids. When $P_{f} \gg P_{\text {grav }}$, the pore pressure built up during impact dominates the dynamics, yielding a quicksandlike or solidlike response depending on the sign of $\Delta \phi$, in agreement 

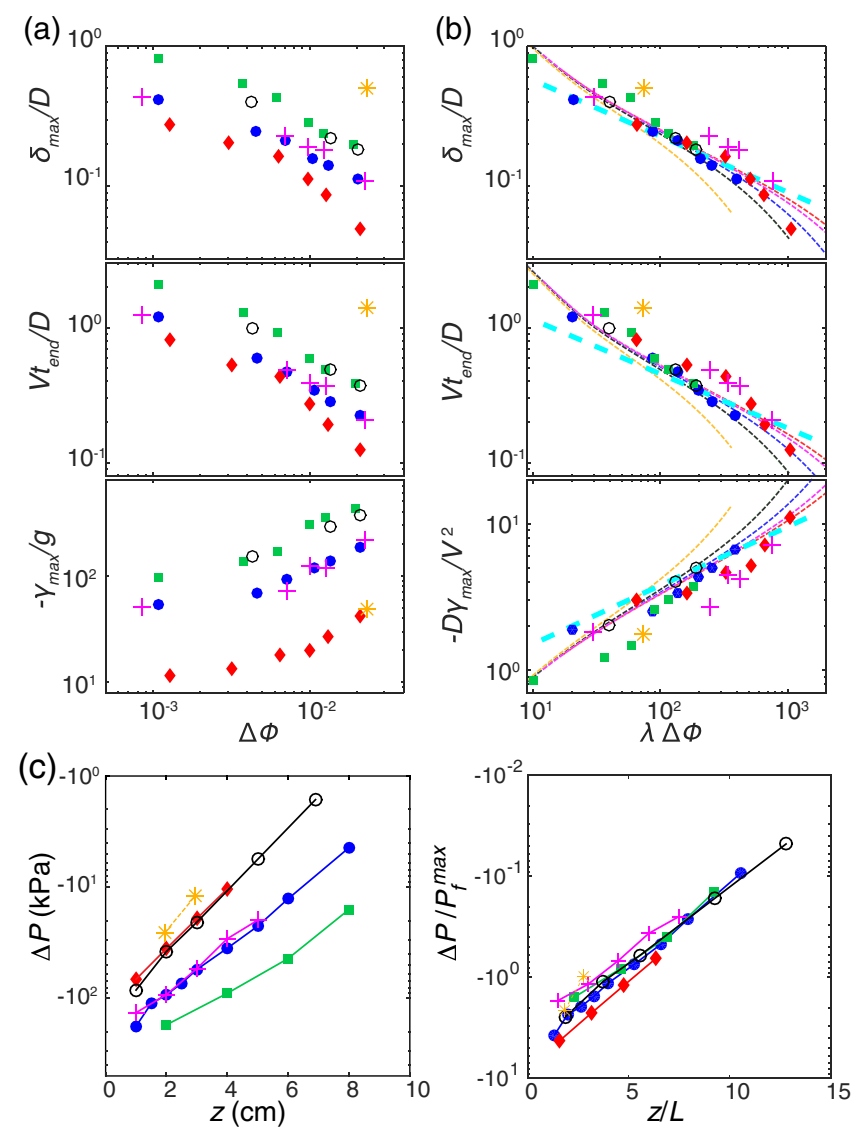

FIG. 3. (a) The maximal indentation $\delta_{\max }$, the typical stopping time $t_{\text {end }}$ (the time at which $\dot{\delta} / V=0.05$ ), and the maximal acceleration $\gamma_{\max }$ plotted against $\Delta \phi$ for different impact speeds (diamonds, $V=1.0 \mathrm{~m} / \mathrm{s}$; disks, $V=2.5 \mathrm{~m} / \mathrm{s}$; squares, $V=5.3 \mathrm{~m} / \mathrm{s}$; with $D=25.2 \mathrm{~mm}$ ), impactor size (circles, $V=3.5 \mathrm{~m} / \mathrm{s}, \quad D=16.5 \mathrm{~mm}$ ) and suspension properties (diamonds, disks, squares, circles, particles $d=170 \mu \mathrm{m}$ in water; crosses, particles $d=500 \mu \mathrm{m}$ in a viscous fluid $\eta_{f}=10.5 \times 10^{-3}$ Pa s; stars, particles $d=500 \mu \mathrm{m}$ in water, with $D=25.2 \mathrm{~mm}$ and $V=2.5 \mathrm{~m} / \mathrm{s}$ ). (b) Rescaled data plotted against $\lambda \Delta \phi$ compared with the predicted scaling laws (thick dashed line) and the improved model (thin dashed lines, see Supplemental Material [29]). (c) Pore-pressure peak measured at different depth and rescaled by the predicted scaling laws: $P_{f}^{\max } \sim$ $\left(\eta_{f} V D \alpha \Delta \phi / \kappa\right)\left(6 A \alpha \eta_{f} D \Delta \phi / \rho_{s} \kappa V\right)^{-1 / 5}$ and $L=a \sim \sqrt{D V t_{m}}$ $(\Delta \phi=0.020$; same legend except for circles, for which $V=2.5 \mathrm{~m} / \mathrm{s}$ and squares, for which $V=5.0 \mathrm{~m} / \mathrm{s}$ ).

with observations $[14,15,17]$. By contrast, when $P_{f} \ll P_{\text {grav }}$ the interstitial fluid contributes negligibly to forces between grains, and so one recovers the classical dry granular case phenomenology $[6,8]$.

Our study thus provides a unifying picture to explain the main regimes observed during the impact of a solid object onto a mixture of grains and fluid, at least when surface tension or cohesive effects can be neglected. It also provides a mechanism to explain the impact-activated solidification observed in more complex shear-thickening

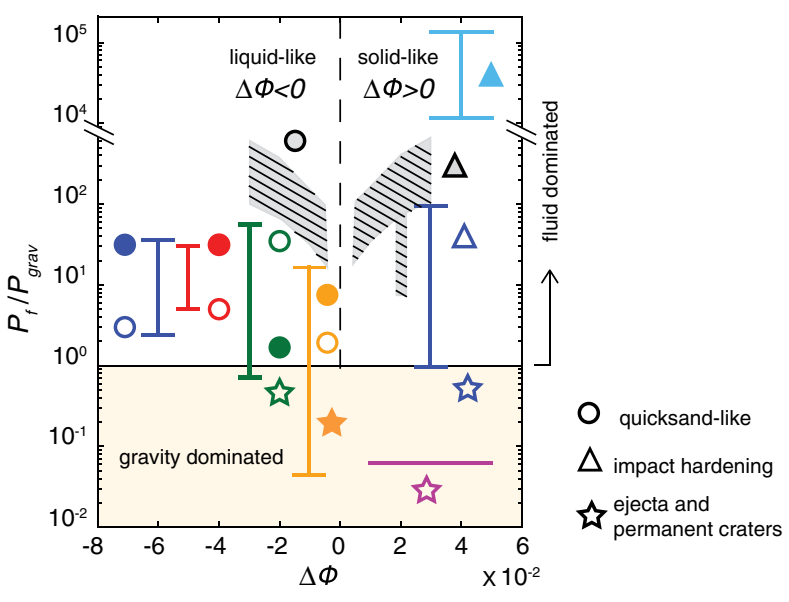

FIG. 4. A unified picture of impact responses in particulate media from dry granular materials to shear-thickening suspensions. Phase diagram $\left(P_{f} / P_{\text {grav }}\right.$ vs $\left.\Delta \phi\right)$ showing diverse impact behaviors (symbols) for a wide range of grain size $(d=10-500 \mu \mathrm{m})$, impact velocity $(V=0.2-300 \mathrm{~m} / \mathrm{s})$, fluid viscosity $\left(\eta_{f}=10^{-5}-10^{-2} \mathrm{Pas}\right)$, and fluid compressibility $\left(1 / \chi=50-2.210^{9} \mathrm{~Pa}\right)$ with data from our work (dense suspensions, the hatched area encompasses all data of Fig. 2 and Fig. 3) and previous studies for glass beads in air [6] (orange), impacts at ultralow air pressure mimicking planetary craters [8] (purple), impacts on fine powders at atmospheric and low air pressure (green [14], red [15], dark blue [17]), impact on suspensions of cornstarch and water [18] (light blue). The vertical bars give the range of $P_{f} / P_{\text {grav }}$ covered in the corresponding study and the horizontal bars give the uncertainty on $\Delta \phi$ (see the table in the Supplemental Material [29]). Symbols indicate the observed behavior for the incompressible (filled) and compressible (open) regime during impact.

media like cornstarch [18]. The key difference between Newtonian and shear-thickening suspensions is that, in the latter, the critical packing fraction $\phi_{c}$ is not a material constant but a decreasing function of the applied stress [23-25]. Therefore, at high impact velocities, the critical packing fraction can drop below the initial packing fraction. The suspension then becomes dilatant and solidifies, due to the pore-pressure feedback mechanism. Since the cornstarch particles are about $10 \mu \mathrm{m}$ and the suspension permeability scale as $\kappa \propto d^{2}$, the DarcyReynolds scaling (2) predicts a 100-fold increases of the pore-pressure compared to that of the Newtonian suspension with glass beads $(d=170 \mu \mathrm{m})$. This is about 1-10 MPa for typical impact conditions, which is in good agreement with impact stress estimations in cornstarch [18] [Fig. 4]. We confirm this viewpoint by performing qualitative measurements of pore pressure in cornstarch suspensions. When an object is impulsively moved in a concentrated suspension of cornstarch in water, pore pressure takes indeed a huge drop (see Fig. S3 in the Supplemental Material [29]). Therefore, it is remarkable that the conjunction of two distinguished mechanisms in 
granular media, the Darcy law and the Reynolds dilatancy, could explain this long-standing puzzle: why we can run on cornstarch.

The authors thank the technical staff of IUSTI and IRPHE for their help in designing the experimental set-up. We also thank Emil Dohlen and students from Polytech'Marseille for preliminary results. This work was supported by the French National Research Agency (ANR) through the Program No. ANR-11-JS09-005-01 and by the Labex MEC (ANR10-LABX-0092) and the A*MIDEX project (ANR-11IDEX-0001-02) funded by the "Investissements d'Avenir" French Government program.

*yoel.forterre@univ-amu.fr

†Present address: Université Claude-Bernard Lyon 1, CNRS, LMFA UMR 5509, F-69622 Villeurbanne Cedex 08, France.

[1] J. C. Ruiz-Suàrez, Rep. Prog. Phys. 76, 066601 (2013).

[2] M. Omidvar, M. Iskander, and S. Bless, Int. J. Impact Eng. 66, 60 (2014).

[3] H.J. Melosh, Impact Cratering: A Geologic Process (Oxford University Press, Oxford, 1989).

[4] N. J. Wagner and J. F. Brady, Phys. Today 62, 27 (2009).

[5] A. H. Clark, A. J. Petersen, L. Kondic, and R. P. Behringer, Phys. Rev. Lett. 114, 144502 (2015).

[6] A. M. Walsh, K. E. Holloway, P. Habdas, and J. R. de Bruyn, Phys. Rev. Lett. 91, 104301 (2003).

[7] J. S. Uehara, M. A. Ambroso, R. P. Ojha, and D. J. Durian, Phys. Rev. Lett. 90, 194301 (2003).

[8] S. Yamamoto, K. Wada, N. Okabe, and T. Matsui, Icarus 183, 215 (2006).

[9] S. Deboeuf, P. Gondret, and M. Rabaud, Phys. Rev. E 79, 041306 (2009).

[10] J. O. Marston, E. Q. Li, and S. T. Thoroddsen, J. Fluid Mech. 704, 5 (2012).

[11] R. Zhao, Q. Zhang, H. Tjugito, and X. Cheng, Proc. Natl. Acad. Sci. U.S.A. 112, 342 (2015).

[12] S. T. Thoroddsen and A. Q. Shen, Phys. Fluids 13, 4 (2001).

[13] D. Lohse, R. Rauhé, R. Bergmann, and D. Van Der Meer, Nature (London) 432, 689 (2004).

[14] J. R. Royer, E. I. Corwin, A. Flior, M.-L. Cordero, M. L. Rivers, P. J. Eng, and H. M. Jaeger, Nat. Phys. 1, 164 (2005).

[15] G. Caballero, R. Bergmann, D. van der Meer, A. Prosperetti, and D. Lohse, Phys. Rev. Lett. 99, 018001 (2007).

[16] J. R. Royer, E. I. Corwin, B. Conyers, A. Flior, M. L. Rivers, P. J. Eng, and H. M. Jaeger Phys. Rev. E 78, 011305 (2008).
[17] J. R. Royer, B. Conyers, E. I. Corwin, P. J. Eng, and H. M. Jaeger, Europhys. Lett. 93, 28008 (2011).

[18] S. R. Waitukaitis and H. M. Jaeger, Nature (London) 487, 205 (2012).

[19] M. Roché, E. Myftiu, M. C. Johnston, P. Kim, and H. A. Stone, Phys. Rev. Lett. 110, 148304 (2013).

[20] R. M. Iverson, Rev. Geophys. 35, 245 (1997).

[21] M. Pailha and O. Pouliquen, J. Fluid Mech. 633, 115 (2009).

[22] B. Andreotti, Y. Forterre, and O. Pouliquen, Granular Media: Between Fluid And Solid (Cambridge University Press, Cambridge, England, 2013).

[23] N. Fernandez, R. Mani, D. Rinaldi, D. Kadau, M. Mosquet, H. Lombois-Burger, J. Cayer-Barrioz, H. J. Herrmann, N. D. Spencer, and L. Isa, Phys. Rev. Lett. 111, 108301 (2013).

[24] R. Seto, R. Mari, J. F. Morris, and M. D. Morton, Phys. Rev. Lett. 111, 218301 (2013).

[25] M. Wyart and M. E. Cates, Phys. Rev. Lett. 112, 098302 (2014).

[26] E. Brown and H. M. Jaeger, Rep. Prog. Phys. 77, 046602 (2014).

[27] A. Fall, F. Bertrand, D. Hautemayou, C. Mezière, P. Moucheront, A. Lemaître, and G. Ovarlez, Phys. Rev. Lett. 114, 098301 (2015).

[28] F. Boyer, E. Guazzelli, and O. Pouliquen, Phys. Rev. Lett. 107, 188301 (2011).

[29] See Supplemental Material at http://link.aps.org/ supplemental/10.1103/PhysRevLett.117.098003 for movie sequences of the experiments, detailed setup description, additional theoretical elements, and additional figures.

[30] O. Reynolds, Philos. Mag. Ser. 5 20, 469 (1885).

[31] The effective weight of the suspension is $\phi_{0} \Delta \rho g z \approx 400 \mathrm{~Pa}$ with $\phi_{0}=0.56, \Delta \rho=\rho_{p}-\rho_{f}=1500 \mathrm{~kg} / \mathrm{m}^{3}$ and $z=5 \mathrm{~cm}$.

[32] R. Jackson, The Dynamics Of Fluidized Particles (Cambridge University Press, Cambridge, England, 2000).

[33] For the suspensions considered in our study, the steady Darcy law can be considered as valid because the impact time $D / V$ is always large compared to the Stokes diffusion time at the pores scale $\kappa /\left(\eta / \rho_{f}\right)$.

[34] S. Roux and F. Radjai, in Physics of Dry Granular Media (Springer, New York, 1998), p. 229.

[35] The Reynolds dilatancy equation is valid in the quasistatic approximation, which is justified here as long as the magnitude of the (negative) pore pressure is high (typically the inertial number $I=\dot{\gamma} d / \sqrt{P / \rho_{p}} \sim 10^{-2}$ with $\dot{\gamma} \sim V / L \sim$ $10^{2} \mathrm{~s}^{-1}$ and $P \sim 10 \mathrm{kPa}$, while the viscous number is $\left.J=\eta \dot{\gamma} / P \sim 10^{-5}\right)$.

[36] A. Schofield and P. Wroth, Critical State Soil Mechanics (Cambridge University Press, Cambridge, England, 1968).

[37] A. Seguin, Y. Bertho, F. Martinez, J. Crassous, and P. Gondret, Phys. Rev. E 87, 012201 (2013). 